(182)

\title{
A Preliminary Investigation of the Habitat Selection of Indian Pangolin (Manis crassicaudata) in a Tropical Lowland Forest in South-West Sri Lanka
}

\author{
M.G.T. Pabasara ${ }^{*}$, P.K.P. Perera ${ }^{1}$, N.P. Dayawansa ${ }^{2}$ \\ ${ }^{1}$ Department of Forestry and Environmental Science, University of Sri Jayewardenepura, Sri Lanka \\ ${ }^{2}$ Department of Zoology, University of Colombo, Sri Lanka \\ *pabasaramgt@gmail.com
}

\begin{abstract}
Indian pangolin (Manis crassicaudata) is a nocturnal, solitary and illusive mammal which has received less scientific attention. M. crassicaudata is listed as Endangered by IUCN and listed in Appendix II of the Convention on International Trade in Endangered Species of Wild Fauna and Flora (CITES). According to the National Red List (2012) of Sri Lanka, $M$. crassicaudata is listed under "Near Threatened" and is strictly protected by the Flora and Fauna Protection Ordinance of Sri Lanka. Despite its conservation importance, very few studies have examined the ecology of Asiatic pangolin species and, there's virtually no autecological studies done on $M$. crassicaudata in Sri Lanka. This lack of reliable information has impaired the accurate assessment of their conservation needs.
\end{abstract}

This study investigated the habitat preference and utilisation of Indian pangolins in Yagirala forest reserve; a tropical lowland forest in southwest Sri Lanka. Four major types of habitats were identified within the study site; secondary natural forest (SF), Pine-dominated forest (PF), Rubber plantations (RP) and Tea-dominated home gardens (TH). Indian pangolin burrows in search of termites to feed, and rests in underground burrows. Hence, presence of pangolins can be determined based on such indirect observations. Evidences for pangolin presence were collected using $50 \times 100 \mathrm{~m}$ belt transects laid in each habitat type. Field surveys from February to September 2015 recorded 29 pangolin burrows, 35 burrowing marks on ground/termite mounds and 9 scratch marks on logs from all habitat types. Habitat Preference Index (HPI) (given by $\mathrm{HPI}=\mathrm{S}_{\mathrm{n}} / \mathrm{A}$ where $\mathrm{S}_{\mathrm{n}}=$ total indirect observations of pangolin activity and $\mathrm{A}=$ area) was used to determine the intensity of pangolin activity in a specific habitat type. Accordingly the highest preference was for Pine-dominated forest and the ranking of preference followed $\mathrm{TH}<\mathrm{RP}<\mathrm{NF}<\mathrm{PF}$. However, Kruskal-Wallis test to compare the pangolin activity in 4 habitat types revealed only a marginal significance $\left(\chi^{2}=7.579, p=0.05\right)$. Key habitat features associated with borrows were further recorded and the preference for a specific habitat feature was determined from the percentage of its occurrence frequencies (where $\mathrm{Po}=$ [frequency of occurrence of a specific habitat feature/total number of burrows observed] $\times 100 \%$ ). Higher Po indicates a greater preference for a specific environmental factor. Accordingly, the environmental factors preferred by Indian pangolins were determined as medium slope $\left(30^{\circ} \sim 60^{\circ}\right)$, low undergrowth $(10 \sim 50 \%)$, dense closure of canopy $(>\sim 70 \%)$ and presence of rock boulders $(83 \%)$. Key ecological factors influencing the habitat selection of pangolins were further explored using Cluster Analysis.

Keywords: Indian Pangolin, Yagirala Forest, Habitat preferences index, Habitat features 